\title{
Laparoscopic resection of tumor recurrence after radical nephrectomy for localized renal cell carcinoma
}

\author{
Lessandro Curcio, Antonio Claudio Cunha, Juan Renteria, Daniel Presto \\ Department of UroOncology, Ipanema Federal Hospital, Rio de Janeiro, RJ, Brazil
}

\begin{abstract}
Introduction: Local recurrence of Renal Cell Carcinoma (RCC) after radical nephrectomy is a rare event. Some known risk factors are: clinical/pathological stage, locorregional disease and lyimph node positivity. Since up to $30-40 \%$ of patients can achieve a disease-free status, we show a case (video) in which we performed a laparoscopic excision of a local RCC, taking advantage of all the well-known benefits of laparoscopy.

Case report: A 56 years old female with a history of open radical nephrectomy two years before was diagnosed with a mass at the time of surveillance CT imaging during follow-up. The suspected local recurrence was $12 \mathrm{~cm}$, and vascularized predominantly by tributaries originating from the iliac vessels. There was no other site of disease (i.e. brain, lung, liver, bones) and laboratory tests were normal. Laparoscopic approach was approached, by inserting 4 trocars ( 2 of 10 and 2 of $5 \mathrm{~mm}$ ) with the patient in the lateral position.

Result: The procedure lasted 130 minutes, with $220 \mathrm{~mL}$ of estimated bleeding; the larger vessels were ligated with polymer clips (Hem-o-lok) and the smaller handled by ultrasonic clamp. The specimen was removed by a small incision below the umbilicus in an appropriate bag. The patient was feed in the first postoperative day and discharged on the third day. Histopathology revealed sarcoma, with a high degree of mitosis, and negative surgical margins. She was referred to medical oncology for adjuvant therapy consideration.

Conclusion: The laparoscopic resection of recurrent tumor should be encouraged in highly selected cases. The minimally invasive method, with its known advantages, especially for more debilitated patients, can be advantageous when applied to suitable cases.
\end{abstract}

\section{ARTICLE INFO}

Available at: www.brazjurol.com.br/videos/may_june_2014/Curcio_431_432video.htm

Int Braz J Urol. 2014; 40 (Video \#7): 431-32

Submitted for publication:

March 20, 2014

Accepted after revision:

May 15, 2014
Correspondence address:

Lessandro Curcio Gonçalves, MD Av das Américas 13554- Bl 02 / 805 Recreio dos Bandeirantes Rio de Janeiro, RJ, 22790-702, Brazil

E-mail: lessandrocg@ig.com.br 


\section{EDITORIAL COMMENT}

The present video by Curcio et al. nicely depicts that the surgical management of locally recurrent renal cell carcinoma (RCC) which was traditionally considered solely suitable for open surgery can now be performed using a laparoscopic approach when applied to highly select cases. The benefits of minimally invasive surgery (MIS) can in consequence be provided to patients in this highly challenging realm of re-operative surgery. It must be highlighted as was mentioned by the authors that such MIS procedures should be offered to only select patients with locally recurrent RCC where no major adjacent organ resection including but not solely encompassing vascular structures anticipated. A fundamental surgical principle applies in that the surgical modality (i.e. pure laparoscopic, robotic, open) should be tailored to the anticipated difficulty of this locally recurrent tumor resection. Lastly, such challenging salvage surgery should only be performed by experienced and highlyskilled laparoendoscopists, who have meticulously reviewed the pre-operative imaging as a roadmap of what this surgical endeavor will likely entail, with the threshold for open surgical conversion being quite low to at no point compromise the therapeutic potential imparted to this procedure.

Philippe E. Spiess, MD Associate Professor

Department of GU Oncology Moffitt Cancer Center Video Section Editor, International Brazilian Journal of Urology 